\title{
Discussing partner abuse: does doctor's gender really matter?
}

\section{Sylvie H Lo Fo Wong ${ }^{a}$, Ank De Jonge ${ }^{a}$, Fred Wester ${ }^{b}$, Saskia S L Mol ${ }^{c}$, Renée R Römkens ${ }^{\mathrm{d}}$ and Toine Lagro-Janssen ${ }^{\mathrm{a}}$}

Lo Fo Wong SH, Jonge AD, Wester F, Mol SSL, Römkens RR and Lagro-Janssen T. Discussing partner abuse: does doctor's gender really matter? Family Practice 2006; 23: 578-586.

Background. There are conflicting findings on the influence of gender on responding to partner abuse.

Objectives. We aimed to explore gender differences in family doctors' views, attitudes, experiences and practices regarding intimate partner abuse against women.

Methods. We used the focus-group method with a stratified, randomized sample of family physicians. Three male and three female groups took part. Two independent researchers analysed the transcripts of the discussions.

Results. There were differences between male and female groups in discussing partner abuse, although similarities were also noted. Major contrasts in opinions were seen in (i) the role of sexuality: part of the male family doctors stated that denial of sexual relationships by a spouse was a contributing and eliciting factor to male aggression, whereas female doctors emphasized unanimously the humiliation of sexual coercion and the danger of opposing. (ii) Children as witnesses: this issue was discussed in female groups only, (iii) female doctors talked about emotional involvement with patients and male doctors about keeping distance, (iv) female doctors viewed leaving an abusive partner as a process whilst male doctors saw no progress, (v) experiences with abused patients: female doctors remembered more actual cases and (vi) practices in managing partner abuse differed between men and women.

Conclusion. These remarkable gender-related differences among doctors might affect care for abused women. Doctors should be aware of gender-related views, attitudes and practices that can be harmful to their patients.

Keywords. Family physician, focus group, gender, intimate partner abuse, women.

\section{Introduction}

There is broad agreement to recognize intimate partner abuse as a serious health concern for women. ${ }^{1}$ Worldwide population surveys among women indicate that between 10 and $50 \%$ were at some stage abused by an intimate partner. ${ }^{2-4}$ Cross-sectional studies in waiting rooms of family doctors in the UK, Ireland and Australia found that $37-41 \%$ of the female patients ever-experienced partner abuse. ${ }^{5-7}$ In general it is a hidden problem in medical practice, as underreporting is almost universal. ${ }^{8,9}$ On the other hand, doctors often refrain from asking because of feelings of powerlessness, inability to offer a useful intervention, scepticism and aversion to the problem. ${ }^{10-16}$ Partner abuse is the sort of problem in which a doctor's attitude is of great importance to help patients disclose and initiate care. ${ }^{9,17}$ Part of the knowledge on a doctor's attitudes comes from focus-group studies that describe how physicians with a special interest in domestic violence identify and treat victims of partner abuse. They emphasize the necessity of an atmosphere that promotes selfdisclosure together with the skill of non-judgemental listening and the need to break through denial. ${ }^{18-20}$

In spite of the recognition of partner abuse as a major problem, as it does not only affect women but

\footnotetext{
Received 11 August 2005; Accepted 8 March 2006.

${ }^{a}$ Department of Family Medicine, Women's Studies Medical Sciences, University Medical Centre St Radboud, Nijmegen, ${ }^{\mathrm{b}}$ Communication Sciences, Radboud University Nijmegen, ${ }^{\mathrm{c}}$ Julius Centre for Health Sciences and Primary Care, University Medical Centre Utrecht, and ${ }^{\mathrm{d}}$ Institute for Security and Crime, University of Tilburg, The Netherlands. Correspondence to Sylvie H Lo Fo Wong, Radboud University Medical Centre, Department of Family Medicine, Women's Studies Medical Sciences, PO Box 9101/HAG 117, 6500 HB Nijmegen, The Netherlands; Email: slofowong@chello.nl
} 
also their children, a gap still remains between the required attention for abused female patients and professional training. ${ }^{21,22}$ Guidelines on management of partner abuse have been released but little is known about their reach, acceptance and implementation in family practice. ${ }^{23-25}$

The ongoing under-identification and lack of attention for abused women calls for a closer look at family doctors' attitudes regarding this problem. In this respect, the recent increase of female family doctors must be taken into account. Research shows female doctors to be more interested in psychosocial problems and female patients to give more psychosocial information to female doctors. ${ }^{26,27}$ Some studies on partner abuse find female doctors to be more involved with victims, showing more commitment and adequate responses compared with male doctors, where others find no effect of gender. ${ }^{11,13,14,28,29}$

Considering these conflicting findings on gender influence and the recent increase of female family doctors, a qualitative study may provide more insight in the role of gender. We therefore conducted a focus-group study to explore the views, attitudes, experiences and practices towards intimate partner abuse and to explore whether gender really matters in discussing these topics.

\section{Methods}

\section{Study design}

This focus-group study was part of an intervention study to evaluate the effects of training on identifying female patients with intimate partner abuse in a randomized controlled trial. In the first part of the study we aimed to explore the views, attitudes, experiences and practices of family doctors towards partner abuse. Topics that emerged from the focus groups were used to design the (intervention) training which took place after the focus-group study (Fig. 1).

Because physicians' attitudes towards partner abuse have not been studied before in The Netherlands, a qualitative research method, focus-group discussion, was chosen to enable in-depth exploration. ${ }^{30}$

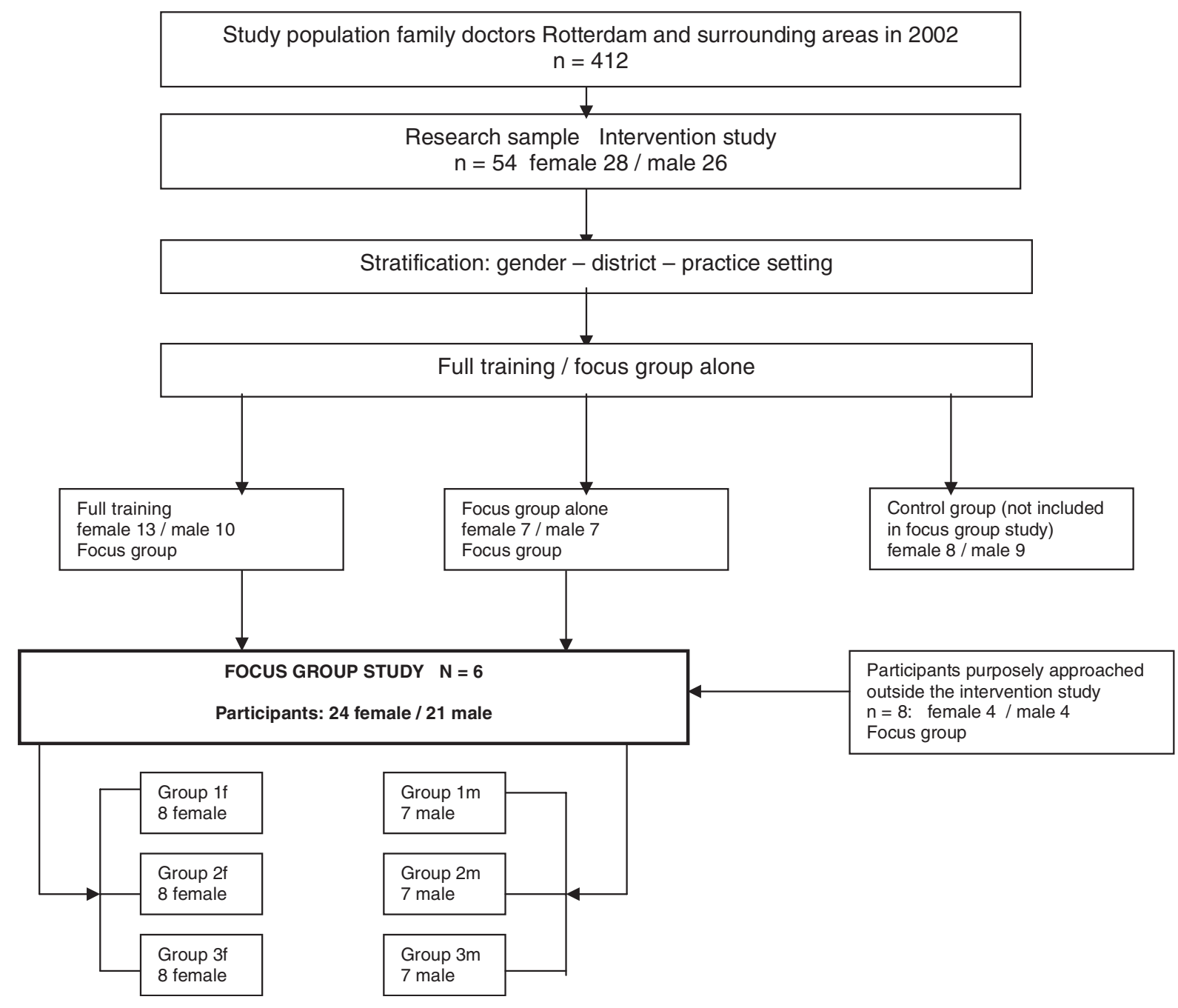

FIGURE 1 Design of the focus-group study 
In October 2002 all family doctors in Rotterdam and its non-urban surroundings $(n=412)$ were invited by letter to participate in the intervention study. Fifty-four family doctors (13\%) agreed to participate. Twenty-six male and twenty-eight female doctors from all types of practice settings, districts and age groups were included in the intervention study. The focus on the influence of gender required a comparable number of male and female doctors. The research sample was divided into strata with similar characteristics: gender, districts and practice setting. From this sample 37 participants were assigned to the focus groups. Because we assumed that participants of the focus-group study were more interested in partner abuse than the average, we purposely approached 'non-responders' until we reached the required number of eight more family doctors for inclusion in the focus groups. The aim was to diminish selection bias and gain information from participants outside the intervention study. We selected the additional participants on the basis of age, gender and residential district. From the resulting 45 doctors we formed 6 focus groups ( 3 male and 3 female).

In view of the sensitivity of the issue as well as our aim to explore gender differences, we conducted exclusively single gender groups to enhance an unhindered exchange of opinions and minimize bias from socially acceptable responses in the discussions. Self-censorship is known as one of the pitfalls of focus groups.

In focus-group studies, information is gathered from group interaction until theoretical saturation takes place. ${ }^{30}$ In studies with homogeneous groups, in general two to four groups are considered enough to reach that point. ${ }^{31,32}$

\section{Data collection and analysis}

A short questionnaire provided demographic data and the participant's estimate of the number of abused female patients encountered in 1 year as well as information on previous education on domestic violence.

A moderator with the same gender as the group conducted the group discussions in February 2003. The two moderators were qualified senior social scientists, familiar with leading groups and with no special interest in domestic violence. The topic guide with eight key questions was developed in discussion with the moderators and tested in a pilot. All questions were used to generate discussion among participants. (See Box 1).

Participants received a small incentive $(€ 40)$ for their effort.

Group discussions lasted 1 hour and a half each and were recorded on audiotape. The research assistant transcribed the tapes. Both the researcher (SLFW) and the assistant (MS) observed the group discussions and took field notes and described non-verbal interactions. The differences between male groups and those
Box 1 Interview guide

1. What images come to mind when you think of intimate partner abuse against women?

2. When thinking of intimate partner abuse of women, what feelings/tendencies do you experience?

3. In intimate partner relationships, what kind of behaviour would you classify as violence and what would you not?

4. In your opinion, which factors can contribute to intimate partner abuse?

5. What difficulties do you encounter when dealing with intimate partner abuse in your office?

6. What are your clinical experiences in this area so far (positive and negative)?

7. What hinders you when you suspect intimate partner abuse in clinical practice?

8. As a family physician, do you think you have a role to play with regard to intimate partner abuse?

between female groups were mainly in the amount of emphasis they placed on certain issues. We did not collect new themes after two groups of each gender.

The first researcher (SLFW) checked the transcripts with the field notes and she and a second researcher (AJ) analysed each transcript.

All comments were sorted per gender, per key question. ${ }^{33}$ This procedure resulted in 16 documents, 8 per gender. The two researchers independently searched for patterns that emerged with each question and subsequently they defined the most important themes together. In accordance with the grounded theory method, specific themes within the groups' narratives were identified first; the transcripts were coded accordingly next. ${ }^{31}$ In case of disagreement, both researchers tried to reach consensus on the influence of gender. In case of a remaining discrepancy, a third researcher (ALJ) read the transcripts with a focused question and the three analysts discussed until agreement was reached. To examine the findings on themes more closely, all transcripts were entered and coded in the ATLAS.ti software program (Visual Qualitative Data Analysis-Management-Model Building-Version WIN 4.2) to compare groups and genders. The study was undertaken with the consent of the ethical committee of the University Medical Centre St Radboud: CMO, region Arnhem, Nijmegen, nr. 2002/275.

\section{Results}

During the purposive sampling of non-responders by telephone, we heard some reasons for non-response such as time-pressure and not viewing partner abuse as a medical issue. In our sample the ratio of male 
TABLE 1 Demographics of focus-group participants and total/study population

\begin{tabular}{|c|c|c|c|c|}
\hline & $\begin{array}{c}\text { Male } \\
(n=21) \\
47 \%\end{array}$ & $\begin{array}{c}\text { Female } \\
(n=24) \\
53 \%\end{array}$ & $\begin{array}{c}\text { Total } \\
(n=45) \\
n(\%)\end{array}$ & $\begin{array}{c}\text { Study } \\
\text { population } \\
\left(n=415^{\mathrm{a}}\right) \\
\text { M } 74 \% / \mathrm{F} 26 \%\end{array}$ \\
\hline \multicolumn{5}{|l|}{ Age groups } \\
\hline$<40$ years & 4 & 6 & $10(22)$ & 13 \\
\hline $40-50$ years & 4 & 12 & $16(36)$ & 43 \\
\hline$>50$ years & 13 & 6 & $19(42)$ & 44 \\
\hline \multicolumn{5}{|l|}{ Practice type ${ }^{b}$} \\
\hline Solo & 6 & 3 & $9(21)$ & 43 \\
\hline Group $^{c}$ & 11 & 13 & $24(53)$ & 42 \\
\hline Health centre $^{\mathrm{d}}$ & 3 & 8 & $11(25)$ & 14 \\
\hline \multicolumn{5}{|l|}{ District type $^{\mathrm{b}}$} \\
\hline Wealthy & 6 & 5 & $11(25)$ & \\
\hline Mixed & 7 & 6 & $13(30)$ & \\
\hline Deprived & 7 & 13 & $20(45)$ & \\
\hline \multicolumn{5}{|l|}{ Full-/part-time } \\
\hline $\mathrm{FT} \geqslant 4$ days & 11 & 3 & $14(31)$ & 77 \\
\hline $\mathrm{PT}<4$ days & 10 & 21 & $31(69)$ & 23 \\
\hline
\end{tabular}

${ }^{a}$ Survey of the District Association of Family Physicians Rotterdam and surroundings 2003.

${ }^{\mathrm{b}}$ Practice type, district type: total number for male doctors does not add up to 21 because 1 participant (a trainee) was not settled.

${ }^{\mathrm{c}}$ Two or more doctors in one family practice.

${ }^{\mathrm{d}}$ Cooperation of family doctors with other primary health care professionals.

to female doctors was $1: 1$; the ratio in the study population is 3:1. Female physicians are younger; they work part-time and in health centres more often.

Each focus group contained a mix of all ages, practice types and districts in order to diminish differences between groups, except for gender. Participants in a group were not from the same practice. For the demographics of the participants see Table 1 .

In all six groups it was the first time that participants discussed their views, attitudes, experiences and practices on partner abuse with colleagues. In the discussions we found several similarities and differences between male and female groups, which we will describe hereafter.

\section{Similarities}

Cultural background was a prominent theme in all groups. Participants explicitly named Turkish, Moroccan and Hindustani-Surinamese ethnic groups as groups with repressive attitudes towards women, together with their supposed legitimized violence as expression of masculinity, as important causes of partner abuse. The doctors mentioned that a lack of social support from the family limited the opportunities to leave a violent relationship, especially in women with arranged marriages. All participants underlined the women's vulnerable positions. Repression and abuse of women in fundamentalist Christian religious communities were also mentioned in this respect.
Inequality of power, the dominant position of men in general, was also viewed as an important reason for women to become a victim or to stay in an abusive relationship.

The powerless attitude in women, manifested as resignation and passivity, was viewed as an important aspect of ongoing violence in relationships. Women's supposed unwillingness to leave their abuser was generally considered incomprehensible and evoked frustration.

Perpetrators' motivations and backgrounds were extensively discussed. In all groups the cause of violent behaviour was seen as the outcome of upbringing, child neglect and abuse, but also as a result of alcohol-, drugabuse and accompanying psychiatric diagnoses.

Feelings of powerlessness of the doctor were considered to be a barrier when partner abuse was suspected. Across groups, inability to solve the problem caused irritation. Most doctors acknowledged that abused women frequently consulted for undefined somatic complaints.

Doctors' fear of perpetrators' aggression as a barrier in addressing partner abuse was mentioned explicitly and comprehensively in all groups. Especially, if a doctor knew about the criminal history of the perpetrator or had experienced violence by some patient before, they would refrain from asking.

The double role of a family doctor giving medical care to both victim and perpetrator was seen as a major barrier in responding to partner abuse. All participants stressed that knowing the background of a perpetrator made it difficult. Understanding perpetrators' motives as well as disbelief, anger and shock were expressed. Fear of making false accusations, causing offence and the risk of losing a patient were serious concerns.

The task of the family doctor was primarily seen as identifying the abuse. The majority of participants in all groups expressed this opinion. Supporting women in abusive relationships was the next objective. Most participants agreed that they were under-identifying abused women. Patients' reluctance to disclose, time constraints and unawareness of the possibility of abuse, were often mentioned in this respect. Two doctors, one male and one female, did not see it as their task to identify abuse and considered that rather as a waste of time.

In conclusion, male and female doctors expressed similar views on major causes of partner abuse against women: the role of culture; power inequality; women's powerless attitude and the role of the perpetrators' childhood experiences. Male and female doctors also agreed on issues regarding asking about or discussing partner abuse: feeling powerless; fear of a perpetrator's aggression and the double role of a family doctor. Participants were almost unanimous in viewing it their task to identify and support victims of partner abuse. 


\section{Differences}

Views on the role of sexuality. In discussing the causes of partner abuse, in one of the male groups the role of sexuality became a central issue. In this male group the denial of sex by a spouse was viewed as a contributing and eliciting factor to male aggression. Some doctors expressed the opinion that women could exercise power by refusing sex and therefore provoke violence.

\section{Q: male group $1 \mathrm{~m}$}

"Sex plays an important role, I have never seen a good sexual relationship go together with abuse" "Let's put it simply, a woman has one means of exercising power and that is simply keeping her legs together and he will have his way..." "It contributes, I think, it plays an important role." (Quotations were translated from Dutch into English.)

"I sometimes explain to the lady: he hits, that's unpleasant, but if you constantly keep your legs together, that's also terribly unpleasant, that's not hitting but just as aggressive sometimes..." "Sometimes explaining that keeping your legs together is also an aggressive act, if she acknowledges this, then at least she knows why the hitting occurs, because it is also a smack..."

There was one doctor with a strongly contrasting opinion in this group:

\section{$Q$ : male group $1 \mathrm{~m}$}

"I think it is just the other way around, the sexual relationship is bad because of a bad personal relationship, and not the other way around"

But opinions in the group did not move to his side. The two other male groups were less explicit in their views on sexuality. However, in one group the acknowledgement of women's rights to set limits in sexual relationships nowadays was accompanied by laughs and jokes. In the other group one doctor made the assumption that sometimes the woman's 'teasing' behaviour provoked male aggression. In response to this remark another participant opposed and considered it a socially unacceptable opinion.

In contrast, the female groups pointed out a different view on the role of sexuality. They emphasized unanimously the humiliation of sexual coercion, the danger of opposing to a partner's sexual demands and a woman's right to set limits in a sexual relationship. In none of the female groups, laughs or jokes accompanied the discussion of this theme.

\section{$Q$ : female group $1 f$}

"I can say something about what I think is normal... about the law... you don't have to put up with everything as a woman..."

"I think coercion, to me violence is ... when there is something with coercion.....coercion to do sexual acts... and when women resist then the hitting starts, that's the process..."

Views on leaving an abusive relationship. Male doctors saw abused women step into abusive relationships time and again. Leaving did not lead to any progress for women and this view was mainly expressed in all male groups.

$Q$ : male group $2 m$

"... there are women that repeatedly come into the same situation, they divorce and choose another partner and then it is often the same..."

"... she leaves and chooses another partner and the next month it's the same all over, the new partner picks up the thread where the other one left off"

In female groups, leaving an abuser was predominantly viewed as a process. Women could learn from their experiences although it would sometimes take time and more than one abusive relationship. The observation that repetition does not only stand for failure was solely heard in the female groups.

Q: female group if

"... it is my experience with several women that it is a process, yes and that more things are needed, patience for instance." "I sometimes see a woman get into the same situation, time after time, but I think, well those things happen and maybe it will take three attempts before she succeeds... it is important that I don't despair."

Emotional involvement with victims. In all female groups, several doctors mentioned that they could manage only a limited number of these cases a day. Female doctors tended to be more emotionally involved with abused patients and reported difficulties in distancing themselves from these problems. As a self-defence mechanism some even decided to block communication when confronted with too many cases.

$Q$ : female group $3 f$

"... I can handle only three of these cases a day, a pity for the fourth one but I simply cannot take more...." "That's even too many."

In all male groups, doctors said they experienced few or no difficulties in discussing abuse with a patient. Avoiding emotional involvement was predominant. If male doctors blocked the communication, they mentioned time constraints as a limiting factor. Involvement was viewed as tricky business with low success rates and therefore the primary reaction was keeping distance.

\section{Q: male group $1 \mathrm{~m}$}

"... I don't want to have anything to do with it" "... because it is dangerous territory ... every step you make you end up in a marsh with quicksand." 
Children as witnesses. As children were not explicitly mentioned in the topic guide, a great difference appeared in the way groups discussed the position of the children. In all female groups, concerns about children witnessing the abuse were raised spontaneously. Doctors discussed and expressed their professional responsibility for the children.

$Q$ : female group $2 f$

“... The children, that bothers me, you don't have good assisting agencies for them ... they risk growing up and becoming perpetrators and victims..."

“... they don't have to be abused themselves ... but they see it happening..."

During the discussion the female doctor that denied having a task in identifying partner abuse modified her opinion at the end of the focus group, because of the children.

In the male groups, children as witnesses were not discussed. Only twice short remarks were made about child abuse as this was seen as a more serious problem than partner abuse and with a more acute need to act.

Experience with partner abuse cases. Female doctors talked in more detail about their experiences with abuse cases, although no numbers were mentioned. They reported to have been confronted with severe cases of abuse, occasionally with deadly consequences.

In male groups, participants mentioned that they hardly came across any cases of abuse although doctors working in deprived districts stated they saw more than those in wealthy areas.

$Q$ : male group $1 \mathrm{~m}$

"... coming here this evening... it was not easy to remember the number of actual cases. I came across six cases in all these (twenty) years...."

Practices in managing partner abuse. Female doctors exchanged strategies on how to respond to abused women, how they actively asked, managed and assessed the safety of their patients. Meanwhile they reflected on their own emotions.

\section{$Q$ : female group $2 f$}

"(I asked)... how safe are you, is it possible to go home, what kind of care is possible ....get someone in the home, finally we made a phone call, I said call from here and ask your friend to come over, or whatever. It didn't work out, then she went home, and in the afternoon the police came when she was shot dead...so this makes it more complicated for me"

In the male groups there were fewer discussions on how actual cases were handled. Theorizing on how to respond and whether questions to identify abuse should be asked or not was the main issue.

\section{$Q$ : male group $2 m$}

"But yes, how often does it occur?" "Maybe more often than we think." (Assenting sounds) "But maybe we don't see it." "Yes exactly, ok, but is there a request for help?" "That is the second thing." "Yes, yes, that is the point." "But if you get a request for help, do you know what you are going to do?" "Well I don't know, it depends on the situation, no..."

There was much agreement about the limited effects of actively questioning a patient about abuse. Male doctors reported they would rather wait until a woman decides to disclose her problem herself, emphasizing the need of a request for help. But if a patient revealed her situation and asked for help, she could count on their support.

In summary, the differences between male and female doctors emerged in their contrasting views on sexuality in abusive relationships, on leaving an abusive partner, the emotional involvement and attention for the plight of the children. Female doctors encountered more cases of partner abuse and addressed this problem more actively than their male counterparts.

\section{Discussion}

Our study supports the assumption that there are gender differences in discussing partner abuse.

The first finding in this context was that the course of the discussion and the expressed views on the role of sexuality were considerably contrasting. These contrasting opinions on the role of sexuality between genders have not been reported to this extent in other studies among doctors. ${ }^{14}$ However a recent survey provided a similar finding concerning opinions on violence against women. The Emancipation Monitor 2004, edited by the Social Cultural Plan Bureau and the Central Bureau for Statistics in The Netherlands, which monitors cultural changes in The Netherlands, found that 32\% of the male respondents held the view that a married (or partnered) man can claim his rights in sexual relationships against $17 \%$ of the female responders. Our study demonstrates a discrepancy between these opinions and the standard in modern society underlining a woman's right to self-determination. Holding women responsible for their abuse because of denying of sex, a view held by a number of male family doctors in our study is harmful for women patients and represents a personal prejudice. ${ }^{23,34}$ Considering the fact that this issue was raised extensively in one of the male groups, we can assume that participants felt safe enough to express this 'politically incorrect' view openly. Further research could explore to what extent these views are common among family doctors in The Netherlands in general and in other modern western countries. A doctor's view on 
sexuality will certainly hinder or facilitate an abused woman in raising the real nature of her problem. In addressing the issue of sexuality when training health care providers, it is necessary to include a substantial part on how to deal with personal prejudices, and to not focus on knowledge of guidelines alone.

The second striking finding is that in the male groups children, as witnesses of abuse, were not discussed. Overlooking the children is also described in one other qualitative study but without its relationship with gender. ${ }^{11}$ Educational programmes on child abuse should address more explicitly the consequences of children witnessing partner abuse. Any course on domestic violence should pay attention to these highly related forms of abuse.

The third differing finding is that female doctors showed more emotional involvement, active questioning and engagement with abused patients while male doctors held more negative views and kept more distance, mainly theorizing on how to respond. One possible explanation is that women, abused by men, are more likely to disclose abuse to a female doctor rather than to trust a male doctor. ${ }^{20}$ This finding is consistent with other studies that report female doctors to be more interested in and more involved with psychosocial problems and female patients giving more information to female doctors. ${ }^{9,26,27}$

Another possible explanation is that in contrast to what was actually said, getting involved with abused women was seen as difficult, resulting in a preference for avoidance. This strategy to cope with difficulties was also mentioned by female doctors. However, male doctors mostly mentioned avoidance as an initial coping strategy and female doctors only resorted to this after having seen several cases on 1 day. The contrasting finding about emotional involvement may be reflected in the difference in number of identified abused patients between male and female doctors. In a questionnaire that was filled in prior to the focus groups, male doctors estimated they identified on average 2.48 abused female patients in 1 year whilst female doctors estimated 3.19. These estimates were not corrected for working hours and female doctors worked predominantly part-time (see Table 1).

The negative side effect of too much emotional involvement is considerable distress, which leads to less availability for problem patients. ${ }^{11}$ Another pitfall for female doctors is that too much identification with a female victim may hinder a professional attitude and performance. The fact remains that female doctors, although working part-time, actually saw more cases and even more severe ones, compared with male doctors. It seems that female doctors' part-time schedules are no obstacle for abused women.

This study adds another new finding. These doctors, working mainly in the multi-ethnic community of Rotterdam, the second largest city in The Netherlands, almost unanimously stated that the repression of women in Turkish, Moroccan and Hindustani-Surinamese groups contributes to partner abuse. Their observation that repression of women is associated with an increased risk of partner violence is recently supported by studies in diverse cultures. ${ }^{35,36}$ Two studies recently examined the effects of gender inequality on intimate partner violence. In their analysis of data from Demographic and Health Surveys, they found that gender inequality and high rates of male patriarchal control significantly increased women's risk of intimate partner violence and unintended pregnancy. A cross-sectional survey on domestic violence in health centres for primary care in Turkey reported a prevalence of $49.5 \%$ of ever-experienced marital violence by female patients, which is higher compared with prevalence studies in Australia, Ireland and the UK (37-41\%) ${ }^{5-7}$ Studying the influence of culture and ethnic background on abuse of women is a recent development. It is now acknowledged that it is necessary to seek for interdisciplinary approaches in research to understand partner violence in its cultural context. ${ }^{35-39}$

Further similar themes that were mentioned in all groups confirm findings from other studies. ${ }^{10,20}$ The view that the woman's unwillingness to leave the abuser is responsible for ongoing abuse ('blaming the victim') and at the same time the acknowledgement of the woman's vulnerable position because of an inequality of power between men and women underlines doctors' ambivalence regarding abused women. Together with powerlessness and problems with caring for victim and perpetrator, the dilemmas and barriers that doctor's experiences are almost universal. ${ }^{11,15,20}$ The view that identification of partner abuse as a waste of time also reflects doctor's powerlessness on this matter is of importance. Finally we wish to highlight the doctors' fear for perpetrators' aggression. For these doctors it appeared to be a major barrier in addressing abuse in their surgery. In the current debate on routinely asking all women, we must keep in mind these barriers. ${ }^{20,40}$

In all six groups the allocation of participants to single gender groups was initially questioned. Doctors are seldom aware of the impact of gender on their attitude and professional performance. All participants accepted the explanation given by the moderator, that a sensitive matter can only be discussed openly in a single gender group.

The strength of this design is the opportunity to explore family doctors' responses to partner abuse and the gender-related nature of some of these. We aimed to diminish bias by performing stratification and randomization and adding participants from outside the intervention study. The main limitation of this study is that most doctors who volunteered and agreed to participate are assumed to be more interested in partner abuse than other doctors. Because of the explorative character and qualitative design of this study, findings cannot be 
generalized to the whole population of family doctors. These findings do not necessarily represent participants' performance in daily practice.

However this study highlights gender-specific views, attitudes and experiences that need more attention both in research and training. It is interesting to theorize on the role of gender-related views and attitudes on the one side and the influence of experience and education on the other. Was it primarily gender socialization that made male doctors focus more on the role of sexuality whilst female doctors were too emotionally involved? It is known from studies on male and female psychological development of identity and gender roles, that primacy of self-determination and competitiveness for men and emotional attachment and relational ties for women are basic. ${ }^{41}$ It is a challenge to search for confirmation or refutation of these findings in future studies. Development of a questionnaire to investigate providers' opinions and attitudes regarding violence against women is one of the options. This could lead to more attention for gender-related provider differences in current domestic violence guidelines. Training should address these specific barriers to achieve acceptance and implementation of guidelines. ${ }^{24,42}$

\section{Conclusion}

Few studies explicitly address the role of gender and in this respect this focus-group study adds new information. Education on partner abuse should provide training experiences that in particular address genderrelated issues in order to overcome personal barriers. For instance male doctors should reflect on the effects of masculine views on sexuality and their reluctance to address the problem whilst female doctors should learn in particular to balance more their emotional involvement and professional attitude. Acknowledging these barriers, the emphasis should lie on the many and various lessons to be learned, both by male and female doctors in order to improve quality of care for victims of partner abuse.

\section{Declaration}

Funding support: this research was supported by a grant from the Theia Foundation, Zilveren Kruis Achmea Health Insurance. (The Netherlands) Project nr 200173.

Ethical approval: the study was undertaken with the consent of the ethical committee of the University Medical Centre St Radboud: Commissie Mensgebonden Onderzoek, region Arnhem, Nijmegen, CMO-nr. 2002/275.

Conflict of interest: none of the six authors has declared a conflict of interest.

\section{Acknowledgements}

We wish to thank all the participating family doctors who shared their views and experiences so openheartedly with us; the moderators Door Hezemans and Ron Alma, for leading the discussion in the focus groups skilfully; the research assistant Margriet Straver, for processing the transcripts.

\section{References}

${ }^{1}$ Watts C, Zimmerman C. Violence against women: global scope and magnitude. Lancet 2002; 359: 1232-1237.

${ }^{2}$ Hageman-White C. European research on the prevalence of violence against women. Violence Against Women 2001; 7: 732-759.

${ }^{3}$ Ellsberg M, Pena R, Herrera A, Liljestrand J, Winkvist A. Candies in hell: women's experiences of violence in Nicaragua. Soc Sci Med 2000; 51: 1595-1610.

${ }^{4}$ Römkens R. Prevalence of wife abuse in the Netherlands. Combining quantitative and qualitative methods. $J$ Interpers Violence 1997; 12: 99-125.

${ }^{5}$ Richardson J, Coid J, Petruckevitch A, Chung WS, Moorey S, Feder G. Identifying domestic violence: cross sectional study in primary care. BMJ 2002; 324: 274.

${ }^{6}$ Hegarty KL, Bush R. Prevalence and associations of partner abuse in women attending general practice: a cross-sectional survey. Aust N Z J Public Health 2002; 26: 437-442.

7 Bradley F, Smith M, Long J, O'Dowd T. Reported frequency of domestic violence: cross sectional survey of women attending general practice. BMJ 2002; 324: 271.

${ }^{8}$ Hamberg K, Johansson EE, Lindgren G. "I was always on guard"an exploration of woman abuse in a group of women with musculoskeletal pain. Fam Pract 1999; 16: 238-244.

${ }^{9}$ Hegarty KL, Taft AJ. Overcoming the barriers to disclosure and inquiry of partner abuse for women attending general practice. Aust N Z J Public Health 2001; 25: 433-437.

${ }^{10}$ Sugg NK, Thompson RS, Thompson DC, Maiuro R, Rivara FP. Domestic violence and primary care. Attitudes, practices, and beliefs. Arch Fam Med 1999; 8: 301-306.

11 Taft A, Broom DH, Legge D. General practitioner management of intimate partner abuse and the whole family: qualitative study. BMJ 2004; 328: 618-620.

12 Ferris LE, Tudiver F. Family physicians' approach to wife abuse: a study of Ontario, Canada, practices. Fam Med 1992; 24: 276-282.

${ }^{13}$ Cohen S, De Vos E, Newberger E. Barriers to physician identification and treatment of family violence: lessons from five communities. Acad Med 1997; 72(Suppl. 1): S19-S25.

${ }^{14}$ Rose K, Saunders D. Nurses' and physicians' attitudes about women abuse: the effects of gender and professional role. Health Care Women Int 1986; 7: 427-438.

15 Sugg NK, Inui T. Primary care physicians' response to domestic violence. Opening Pandora's box. JAMA 1992; 267: 3157-3160.

16 van den Akker M, Mol SS, Metsemakers JF, Dinant GJ, Knottnerus JA. Barriers in the care of patients who have experienced a traumatic event: the perspective of general practice. Fam Pract 2001; 18: 214-216.

17 Gerbert B, Johnston K, Caspers N, Bleecker T, Woods A, Rosenbaum A. Experiences of battered women in health care settings: a qualitative study. Women Health 1996; 24: 1-17.

${ }^{18}$ Gerbert B, Caspers N, Bronstone A, Moe J, Abercrombie P. A qualitative analysis of how physicians with expertise in domestic violence approach the identification of victims. Ann Intern Med 1999; 131: 578-584.

${ }^{19}$ Gerbert B, Caspers N, Milliken N, Berlin M, Bronstone A, Moe J. Interventions that help victims of domestic violence. 
A qualitative analysis of physicians' experiences. J Fam Pract 2000; 49: 889-895.

${ }^{20}$ Brown JB, Lent B, Sas G. Identifying and treating wife abuse. J Fam Pract 1993; 36: 185-191.

${ }^{21}$ Ramsay J, Richardson J, Carter YH, Davidson LL, Feder G. Should health professionals screen women for domestic violence? Systematic review BMJ 2002; 325: 314.

${ }^{22}$ Kernic MA, Wolf ME, Holt VL, McKnight B, Huebner CE, Rivara FP. Behavioural problems among children whose mothers are abused by an intimate partner. Child Abuse Negl 2003; 27: 1231-1246.

23 American Medical Association. American Medical Association diagnostic and treatment guidelines on domestic violence. Arch Fam Med 1992; 1: 39-47.

${ }^{24}$ Ferris LE, Norton PG, Dunn EV, Gort EH, Degani N. Guidelines for managing domestic abuse when male and female partners are patients of the same physician. The Delphi Panel and the Consulting Group. JAMA 1997; 278: 851-857.

25 Dienemann J, Campbell J, Wiederhorn N, Laughon K, Jordan E. A critical pathway for intimate partner violence across the continuum of care. J Obstet Gynecol Neonatal Nurs 2003; 32: 594-603.

${ }^{26}$ Kerssens JJ, Bensing JM, Andela MG. Patient preference for genders of health professionals. Soc Sci Med 1997; 44: $1531-1540$.

${ }^{27}$ Hall JA, Roter DL. Do patients talk differently to male and female physicians? A meta-analytic review Patient Educ Couns 2002; 48: $217-224$.

${ }^{28}$ Rodriguez MA, Bauer HM, McLoughlin E, Grumbach K. Screening and intervention for intimate partner abuse: practices and attitudes of primary care physicians. JAMA 1999; 282: 468-474.

${ }^{29}$ Easteal PW, Easteal S. Attitudes and practices of doctors toward spouse assault victims: an Australian study. Violence Vict 1992 7: $217-228$.
${ }^{30}$ Kitzinger J. Qualitative Research: introducing focus groups. BMJ 1995; 311: 299-302.

31 Strauss A, Corbin J. Basics of Qualitative Research; Techniques and Procedures for Developing Grounded Theory. London: Sage Publications; 1998.

32 Morgan D. Planning Focus Groups. Focus Group Kit, Vol. 2. London/New Delhi: Sage Publications; 1998.

${ }^{33}$ Krueger R. Analyzing \& Reporting Focus Group Results. Focus Group Kit. Vol. 6. London/New Delhi: Sage Publications; 1998.

34 Buel SM. Treatment guidelines for healthcare providers' interventions with domestic violence victims: experience from the USA. Int J Gynaecol Obstet 2002; 78 (Suppl. 1): S39-S44.

35 Pallitto CC, O'Campo P. Community level effects of gender inequality on intimate partner violence and unintended pregnancy in Colombia: testing the feminist perspective. Soc Sci Med 2005; 60: 2205-2216.

${ }^{36}$ Gage AJ. Women's experience of intimate partner violence in Haiti. Soc Sci Med 2005; 61: 343-364.

${ }^{37}$ Kasturirangan A, Krishnan S, Riger S. The impact of culture and minority status on women's experience of domestic violence. Trauma Violence Abuse 2004; 5: 318-332.

38 Alper Z, Ergin N, Selimoglu K, Bilgel N. Domestic violence: a study among a group of Turkish women. Eur J Gen Pract 2005; 11: 48-54.

39 Bent-Goodley TB. Culture and domestic violence: transforming knowledge development. J Interpers Violence 2005; 20: 195-203.

40 Taket A, Wathen CN, MacMillan H. Should health professionals screen all women for domestic violence? PLoS Med 2004; 1: 7-10.

${ }^{41}$ Seeman MV. Gender and Pschychopathology, 1st edn. Washington: American Psychiatric Press, Inc.; 1995.

${ }^{42}$ Warshaw C. Intimate partner abuse: developing a framework for change in medical education. Acad Med 1997; 72 (Suppl. 1): S26-S37. 